\title{
Construction, Expression, and Characterization of Thymosin Alpha 1 Tandem Repeats in Escherichia coli
}

\author{
Xiao-Chang Xue, Zhen Yan, Wei-Na Li, Meng Li, Xin Qin, Cun Zhang, Qiang Hao, \\ Zeng-Lu Wang, Ning Zhao, Wei Zhang, and Ying-Qi Zhang
}

State Key Laboratory of Cancer Biology, Department of Biopharmaceutics, School of Pharmacy, Fourth Military Medical University, Xian 710032, China

Correspondence should be addressed to Ying-Qi Zhang; xue_xiaochang@yahoo.com

Received 11 June 2012; Revised 26 November 2012; Accepted 26 November 2012

Academic Editor: Fabio Ferreira Perazzo

Copyright (C) 2013 Xiao-Chang Xue et al. This is an open access article distributed under the Creative Commons Attribution License, which permits unrestricted use, distribution, and reproduction in any medium, provided the original work is properly cited.

\begin{abstract}
Thymosin alpha $1(\mathrm{~T} \alpha 1)$, which is composed of 28 amino acids, has been commercialized worldwide for its immune-modulatory and antitumor effects. T $\alpha 1$ can stimulate T cell proliferation and differentiation from bone marrow stem cells, augment cell-mediated immune responses, and regulate homeostasis of immune system. In this study, we developed a novel strategy to produce T $\alpha 1$

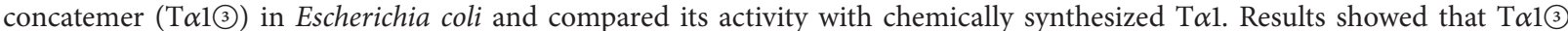
can more effectively stimulate $\mathrm{T}$ cell proliferation and significantly upregulate IL-2 receptor expression. We concluded that the expression system for T $\alpha 1$ concatemer was constructed successfully, which could serve as an efficient tool for the production of large quantities of the active protein.
\end{abstract}

\section{Introduction}

The tandem repeats of proteins and peptides are studied widely and formidable progress has been made in this field. It was reported that tandem amino acid repeats have many functions of stabilizing proteins [1], maintaining conformation [2], elevating activity, and increasing half-life of proteins in blood or tissues. Frasch and colleagues suggested that tandem repeats present in Trypanosoma cruzi transsialidase stabilized the catalytic activity. In addition, repeats present on T. cruzi shed proteins increased trans-sialidase half-life in blood from 7 to almost $35 \mathrm{~h}$ [3]. Some proteins that contain tandemly repeated sequences play important roles in cell membrane skeleton system $[4,5]$.

Thymosin alphal ( $\mathrm{T} \alpha 1)$ is a heat-stable, acidic polypeptide composed of 28 amino acid residues blocked at the Nterminus by an acetyl group [6, 7]. It is an immune modifier that has been shown to trigger lymphocytes maturation, augment $\mathrm{T}$ cell function, induce T-cell differentiation, and promote reconstitution of immune defects. All these findings showed that $T \alpha 1$ could be a useful restorative therapeutic agent in the treatment of immunodeficiency diseases and immunosuppressed conditions [8-10].
In this study, T $\alpha 11^{3}$ which was composed of three repeated copies of $\mathrm{T} \alpha 1$ was fuse-expressed with thioredoxin (trx) in E. coli TOP10 strain and purified by heat treatment and Q-Sepharose Fast Flow ion-exchange chromatography. Then, $\mathrm{T} \alpha 1$ (3) was released by treatment with $0.5 \mathrm{M}$ Cyanogen bromide $(\mathrm{CNBr})$ and purified by SP-Sepharose Fast Flow chromatography. In our strategy, trx acts as a chaperon to help T $\alpha 13$ folding and $\mathrm{CNBr}$ treatment removed any exogenous amino acid (such as Met at the $\mathrm{N}$-terminus for translation start) from $\mathrm{T} \alpha 13$ molecule. So we can get the "natural" T $\alpha 13$. Finally, the biological activity of T $\alpha 13$ on $T$ lymphocyte proliferation and IL-2R expression was assessed.

\section{Materials and Methods}

2.1. Materials. Restriction enzymes, Taq DNA polymerase, and T4 DNA ligase were purchased from TaKaRa. Expression vector pThioHisA and E. coli strain TOP10 (F-mcrA $\Delta$ (mrrhsd RMS-mcrBC) $\varphi 80$ lacZ $\Delta$ M15 $\Delta$ lacX74 recA1 ara $\Delta 139 \Delta$ (ara-leu)7697 galU galK rpsL (Strr)endA1 nupG) were from Invitrogen. DNA fragments were synthesized in BIOASIA. Synthetic T $\alpha 1$ (ZADAXIN) was from Sciclone Pharmaceuticals, USA. The anti-T $\alpha 1$ antibody (ab55635) was purchased 


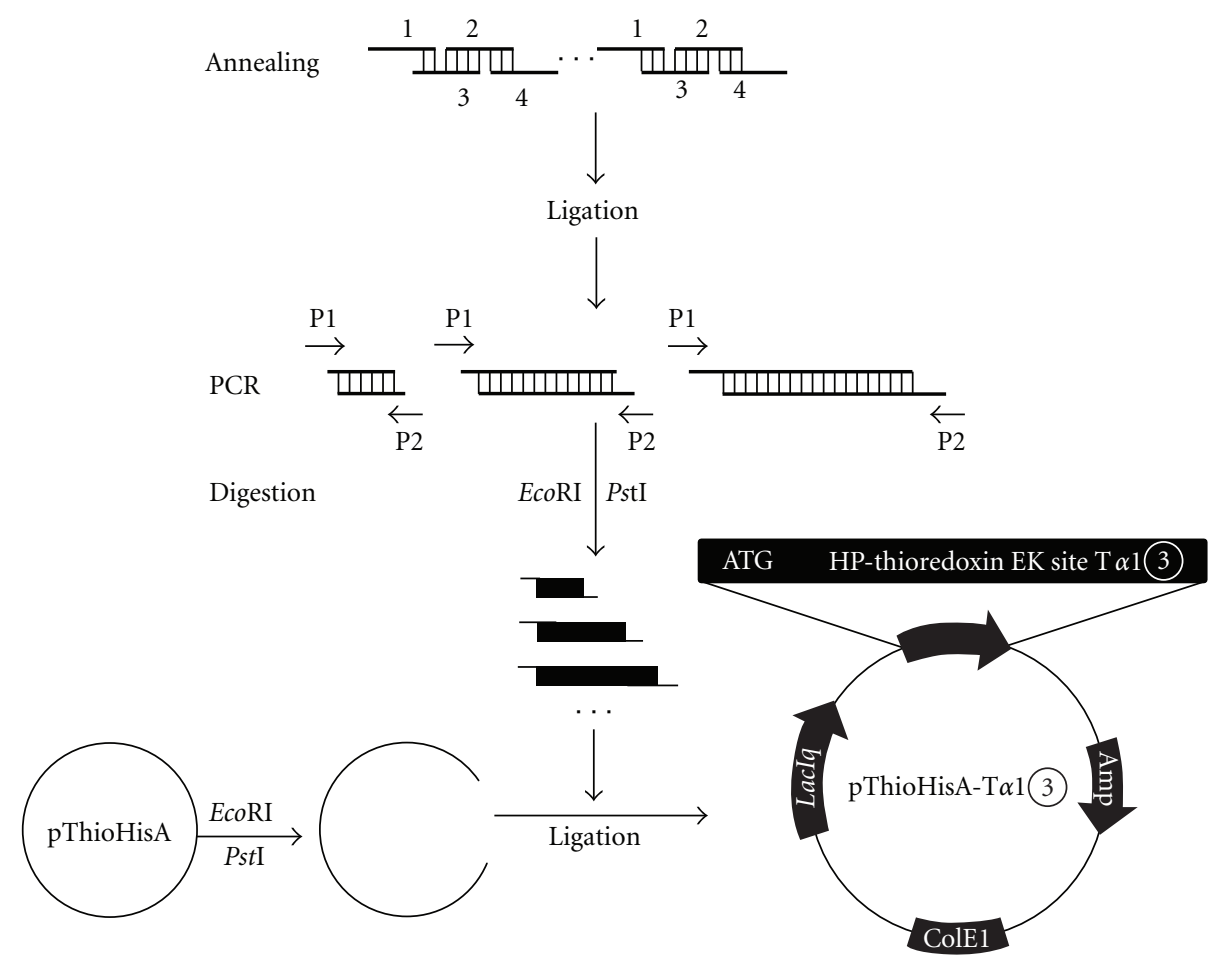

Figure 1: Schematic diagram shows the strategy for constructing T $\alpha 1$ concatemers. The Arabic numerals 1 to 4 are sequences 1 to 4 split from T $\alpha 1$ described previously for concatemers assembly. P1 and P2 are forward and reverse primers for PCR.

from Abcam and FITC-anti-IL-2R $\beta$ (18344D 554452) was from BD Pharmingen.

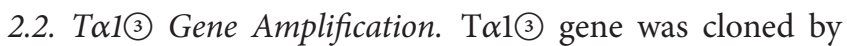
gene synthesis and PCR (Figure 1). The forward primer (with an introduced EcoR I site) was pl: $5^{\prime}$-GGAATTCATGTCTGATGCAGCCGTGGACACCAGCAGCG-3' and the reverse primer (with an introduced Pst I site) was p2: $5^{\prime}$ GCACTGCAGTCAGTTCTGGGCCTCCTCCACCACCT$3^{\prime}$. The template for PCR was annealing products of 4 synthesized fragments listed in Table 1. PCR product was cloned into pGEM-3Zf plasmid to obtain vector pGEMT $\alpha 13$ for identification.

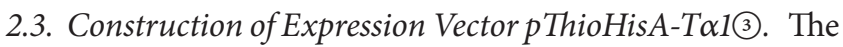
vector pGEM-T $\alpha 13$ was digested with EcoR I and Pst I and cloned into expression vector pThioHisA digested with the same enzymes. The candidate plasmid pThioHisA-T $\alpha 13$ was then confirmed by restriction enzymes digestion and DNA sequencing.

2.4. Expression of the Fusion Protein. The plasmid pThioHisA-T $\alpha 1$ (3) was transformed into E. coli TOP10 strain. A single colony was inoculated into $10 \mathrm{~mL}$ Luria-Bertani (LB) medium supplemented with ampicillin $(100 \mu \mathrm{g} / \mathrm{mL})$ and grown at $200 \mathrm{rpm}$ and $37^{\circ} \mathrm{C}$ overnight. Then it was inoculated into 300 $\mathrm{mL}$ fresh $\mathrm{LB}$ medium in a $500 \mathrm{~mL}$ shake flask and cultured until the OD600 reached 0.5. Trx-T $\alpha 1$ (3) expression was induced by $1 \mathrm{mM}$ IPTG (final concentration) for $4 \mathrm{~h}$. Large
TABLE 1: Nucleotide sequence of DNA fragments split from T $\alpha 1$ for concatemers assembly.

\begin{tabular}{ll}
\hline Number nucleotide sequence \\
\hline 1 & GACACCAGCAGCGAGATCACCACCAAGGACC \\
2 & GGAAGGAGAAGAAGGAGGTGGTG \\
3 & CTTGGTGGTGATCTCGCTGCTGGTGTCCACGG \\
& CGGCGTCGCT \\
4 & GTTCTCGGCCTCCTCCACCACCTCCTTCTTCTCC \\
\hline
\end{tabular}

scale fed-batch culture was performed in a 5-L fermentor as previously described [11].

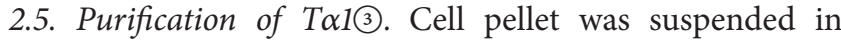
$20 \mathrm{mM}$ Tris/HCl buffer ( $\mathrm{pH}$ 8.0) in proportion of $200 \mathrm{~g} / \mathrm{L}$ and disrupted by sonication. Then, the lysate was incubated at $80^{\circ} \mathrm{C}$ for $10 \mathrm{~min}$ (shaken once every 2-3 min) and cooled quickly. Samples were centrifuged at $12000 \mathrm{~g}$ for $20 \mathrm{~min}$ and the supernatant was loaded onto a Q-Sepharose Fast Flow chromatography column and eluted with linear $\mathrm{NaCl}$ gradient. The purified $\operatorname{Trx}-\mathrm{T} \alpha 13$ was then cleaved by $\mathrm{CNBr}$ $(0.5 \mathrm{M})$ in $70 \%$ formic acid for $24 \mathrm{~h}$. The cleavage reaction was stopped by addition of ten times amount of $\mathrm{H}_{2} \mathrm{O}$ [12] and T $\alpha 13$ was purified by SP-Sepharose Fast Flow chromatography. Purified $\mathrm{T} \alpha 13$ was dialyzed against PBS for later use. 


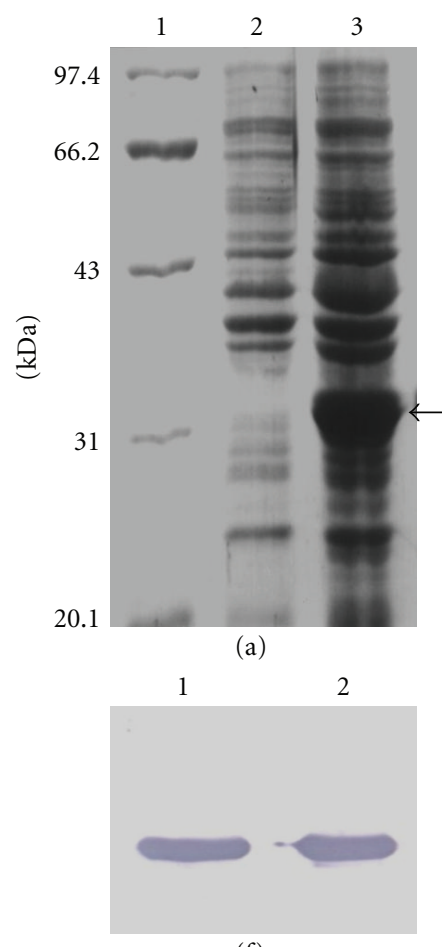

(f)

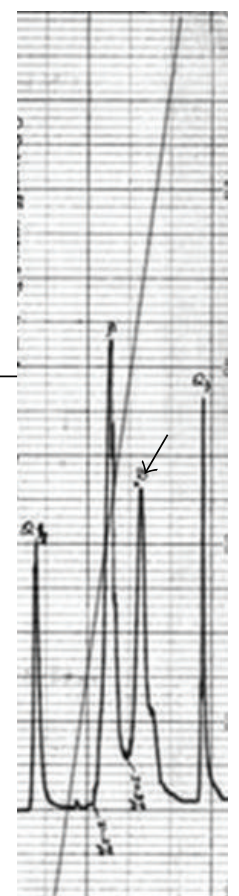

(b)

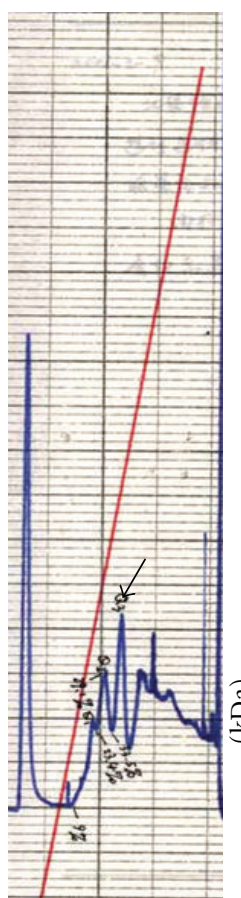

(c)

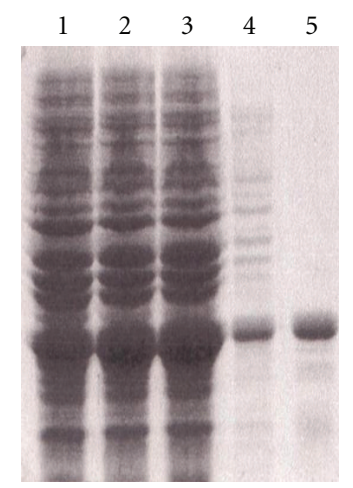

(d)

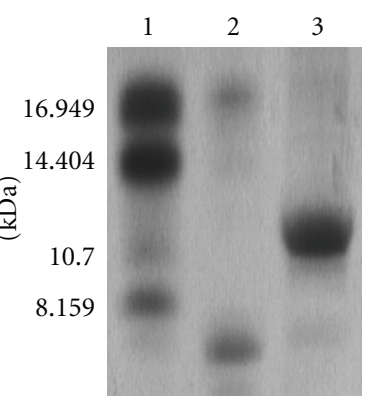

(e)

FIgURE 2: Expression, purification, and identification of T $\alpha 13$. (a) Expression of trx-T $\alpha 13$ in TOP10. Lane 1: protein marker; lane 2: total bacterial proteins of pThioHisA-T $\alpha 13 / \mathrm{TOP} 10$ without induction; lane 3: total bacterial protein with IPTG induction. (b) Chromatogram of Q-Sepharose Fast Flow chromatography for purification of trx-T $\alpha 1$ 3. The arrow indicated trx-T $\alpha 1$ (3). (c) Chromatogram of SP-Sepharose Fast Flow chromatography for purification of T $\alpha 1$ (3). The arrow indicated T $\alpha 1$ (3). (d) SDS-PAGE analysis of trx-T $\alpha 1$ (3) purification. Lane 1-3: total proteins of pThioHisA-T $\alpha 13 / \mathrm{TOP} 10$ after IPTG induction $(1 \mathrm{~h}, 3 \mathrm{~h}, 5 \mathrm{~h})$; lane 4: supernatant of lysate heated at $80^{\circ} \mathrm{C}$ for $10 \mathrm{~min}$; lane 5 : purified trx-T $\alpha 13$. (e) Tricine-SDS-PAGE analysis of T $\alpha 13$ purification. Lane 1: standard peptide marker; lane 2: cleavage products without $\mathrm{T} \alpha$ 13; lane 3: purified T $\alpha 1$ (3). (f) Western-blot analysis of trx-T $\alpha 1$ (3). Lane 1: total bacterial proteins after IPTG induction; lane 2: purified $\operatorname{trx}-\mathrm{T} \alpha 13$.

2.6. Western-Blot Analysis. Proteins were transferred to nitrocellulose membranes $(0.22 \mu \mathrm{m}$, Invitrogen) after SDSPAGE using a Bio-Rad Semi-Dry electrophoretic cell. Western blot analyses were carried out using a T $\alpha 1$ specific antibody and followed by a phosphatase-conjugated goat anti-mouse IgG (Boster, China). Western Blue Stabilized Substrate (Promega) for alkaline phosphatase was used for visualization.

2.7. Biological Activity Assay. The proliferation response of splenocytes was determined by MTT assay. Spleens from C57BL6 mice were dispersed through nylon mesh to generate a single-cell suspension. Then lymphocytes were separated by EZ-Sep 1× Lymphocyte Separation Medium (DKW33-R0100, Dakewe Biotech Company, China) and suspended at $4 \times$ $10^{6} / \mathrm{mL}$ in RPMI 1640 media. For proliferation assay, cells were seeded in 96-well plates $\left(4 \times 10^{5} /\right.$ well $)$ and cultured in the presence of $2.5 \mu \mathrm{g} / \mathrm{mL}$ concanavalin A (ConA) at $37^{\circ} \mathrm{C}$ in $5 \% \mathrm{CO}_{2}$ in humid air. Six h later, $90 \mu \mathrm{L}$ of $\mathrm{T} \alpha 13$ diluted with RPMI 1640 media was added to all but the control wells. The synthetic T $\alpha 1$ and media were used as positive and negative controls. After $66 \mathrm{~h}$ incubation, $20 \mu \mathrm{L}$ of MTT $(0.5 \mathrm{mg} / \mathrm{mL})$ solution was added and the plates were centrifuged $\left(2000 \mathrm{rpm}, 25^{\circ} \mathrm{C}, 10 \mathrm{~min}\right) 4 \mathrm{~h}$ later. Supernatants were discarded, and $100 \mu \mathrm{L}$ of DMSO was added. After incubated at room temperature for $10 \mathrm{~min}$, the solubilized reduced MTT was measured at $570 \mathrm{~nm}$ using a Bio-Rad plate reader and the optical densities were used for calculate growth rate with the formula

$$
\text { Growth rate }(\%)=\frac{\text { OD sample }}{\text { OD control }} \times 100 \% \text {. }
$$

To evaluate the effect of T $\alpha 13$ on the expression level of IL-2R on $\mathrm{T}$ lymphocytes, cells were isolated as before and cultured in the presence of ConA and T $\alpha 13$. The synthetic $\mathrm{T} \alpha 1$ and a recombinant $\mathrm{T} \alpha 1$ monomer prepared in our lab were used as positive controls. Cells were collected and stained $48 \mathrm{~h}$ later according to standard protocol. In brief, $5 \times$ $10^{5}$ cells were washed with PBS and stained in "FACS buffer" (PBS with $0.1 \%$ sodium azide, $2 \%$ FBS, and $1 \mu \mathrm{M}$ EDTA) with FITC-anti-mIL-2R $\beta$ for $10 \mathrm{~min}$ at room temperature. After washing, cells were fixed for 30 minutes on ice with $4 \%$ paraformaldehyde and analyzed on a FACSCalibur flow cytometer (BD Biosciences). 


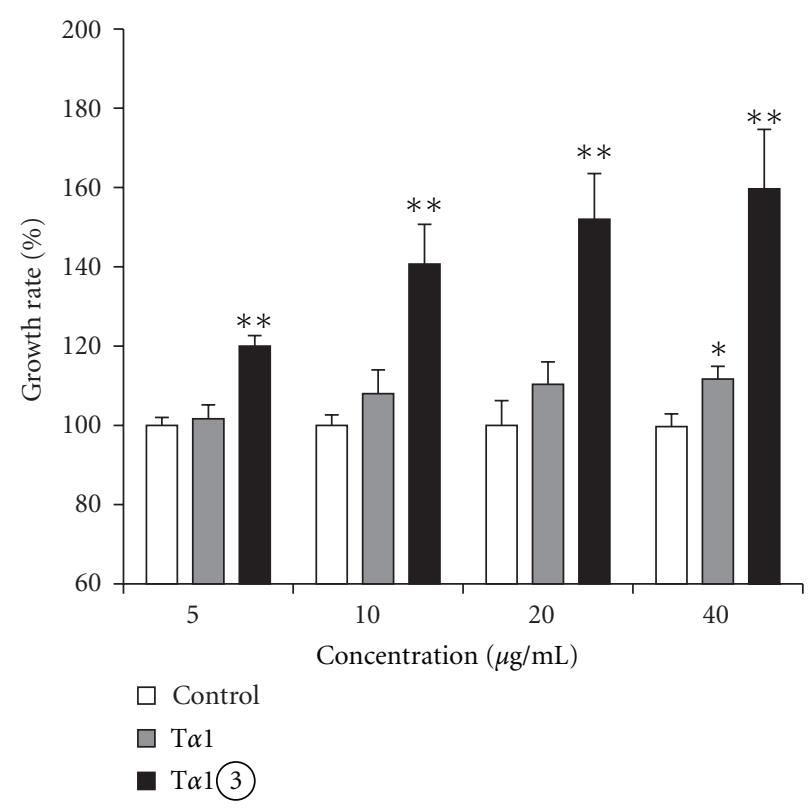

FIGURE 3: T $\alpha 1$ (3) stimulated proliferation of mouse spleen lymphocytes. T lymphocytes from B6 mice spleen were treated with ConA (control) or ConA plus $\mathrm{T} \alpha 13$ or synthetic T $\alpha 1$. Cell proliferation was determined by the MTT viability assay. The assays were repeated in triplicate. $\left({ }^{*} P<0.05\right.$ compared with control group. ${ }^{* *} P<0.05$ compared with $\mathrm{T} \alpha 1$ group. $)$

\section{Results and Discussion}

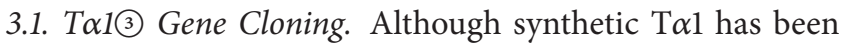
successfully applied in clinical trials for immunodeficiency diseases therapy, the high costs is still a hard nut to crack. Fortunately, molecular biology techniques allowed us to produce recombinant $\mathrm{T} \alpha 1$ in E. coli. Considering that $\mathrm{T} \alpha 1$ is too small to be directly expressed in E. coli, it was usually assembled as concatemers. But some exogenous amino acid residues such as His6 tag or methionine (Met) introduced by the initiation codon AUG usually affects the effect of concatemers [13].

In order to produce the real "natural" concatemers of T $\alpha 1$, we put forward a new strategy as showed in Figure 1. By this strategy, we obtained a series of T $\alpha 1$ concatemers in which T $\alpha 13$ that was assembled by three repeated copies of T $\alpha 1$ gene owned highest proportion. After cloning into pGEM$3 Z \mathrm{f}$ vector, the gene was proven by enzyme digestion and DNA sequencing. The sequence of T $\alpha 1$ (3) gene was consistent with our design as follows: $5^{\prime}$-atgagcgacgccgccgtggacaccagcagcgagatcaccaccaaggaccggaaggagaagaaggaggtggtggaggaggccgagaacagcgacgccgccgtggacaccagcagcgagatcaccaccaaggaccggaaggagaagaaggaggtggtggaggaggccgagaacagcgacgccgccgtggacaccagcagcgagatcaccaccaaggaccggaaggagaagaaggaggtggtggaggaggccgagaactga- $3^{\prime}$.

3.2. Expression of Recombinant Fusion Protein. Both SDSPAGE (Figure 2(a)) and Western blot (Figure 2(f)) analyses of the induced supernatant from pThioHisA-T $\alpha 13 /$ TOP10 showed that a new $31 \mathrm{kDa}$ protein which can be specifically recognized by $\mathrm{T} \alpha 1$ antibody was produced. It suggested that $\operatorname{trx}-\mathrm{T} \alpha 13$ was successfully expressed. Trx was used as a chaperon to guarantee the correct folding of T $\alpha 13$ and trx$\mathrm{T} \alpha 13$ was expressed as a soluble fusion protein.

3.3. Purification of T $\alpha 13$. Both trx and T $\alpha 1$ are heat-stable proteins, so trx-T $\alpha 1$ (3) was easily purified by one-step QSepharose Fast Flow chromatography after the lysate of recombinant bacterial cells was heated at $80^{\circ} \mathrm{C}$ for $10 \mathrm{~min}$ (Figures 2(b) and 2(d)). Then, the purified trx-T $\alpha 13$ was cleaved by $\mathrm{CNBr}$, and $\mathrm{T} \alpha 13$ was purified by SP-Sepharose Fast Flow chromatography (Figures 2(c) and 2(e)). 2LTricine-SDS-PAGE [14] and HPLC analyses were used to identify the purity of $\mathrm{T} \alpha 13$. $\mathrm{CNBr}$ treatment was utilized here to remove the redundant Met from the N-terminus of T $\alpha 13$ to obtain the real "natural" molecule.

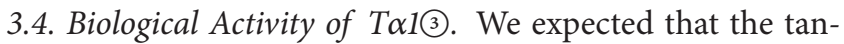
dem repeats could obtain stronger activity through elongating the half-life of $\mathrm{T} \alpha 1$ and simulating polymerization of monomer molecules and thereafter triggering the polymerization and activation of receptors which was usually used by molecules to gain function.

To examine the effect of T $\alpha 1$ (3) on stimulating the proliferation of splenic lymphocytes, we compared the proliferation ratio of mice lymphocytes treated with synthetic T $\alpha 1$ ZADAXIN and T $\alpha 13$. MTT assay results showed that $40 \mu \mathrm{g} / \mathrm{mL}$ synthetic $\mathrm{T} \alpha 1$ could induce significant proliferation of lymphocytes compared to the control $(P<0.05)$, whereas $5 \mu \mathrm{g} / \mathrm{mL}$ T $\alpha 13$ could induce significant proliferation $(P<$ 0.05). Furthermore, the effect of $10 \mu \mathrm{g} / \mathrm{mL}$ T $\alpha 13$ ) was stronger than that of $40 \mu \mathrm{g} / \mathrm{mL}$ synthetic T $\alpha 1$ (Figure 3 ).

In addition, the upregulation of IL-2R on lymphocytes by ZADAXIN purified recombinant T $\alpha 1$ monomer and $\mathrm{T} \alpha 13$ was compared. Results showed that when costimulated with 


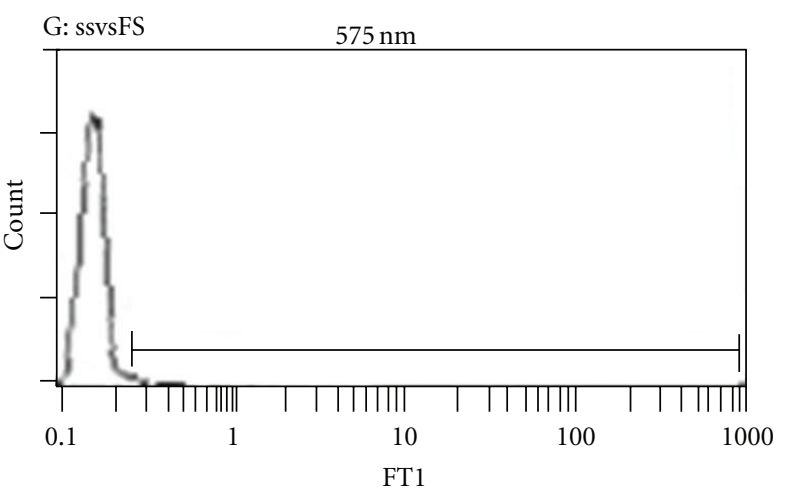

(a)

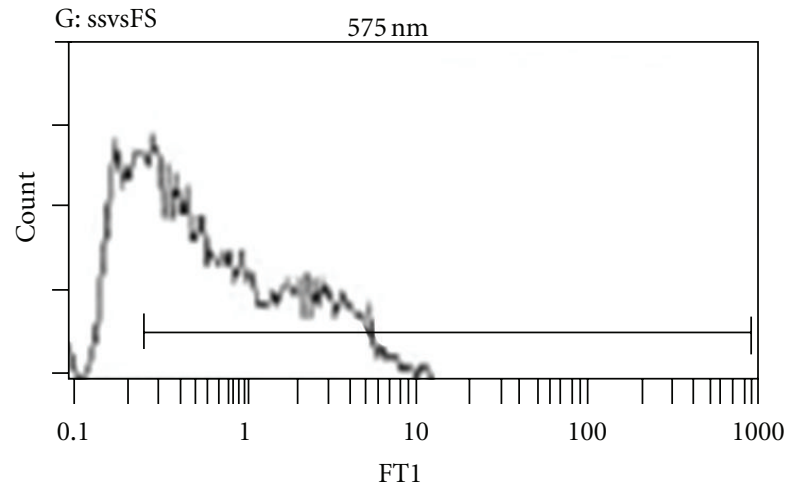

(c)

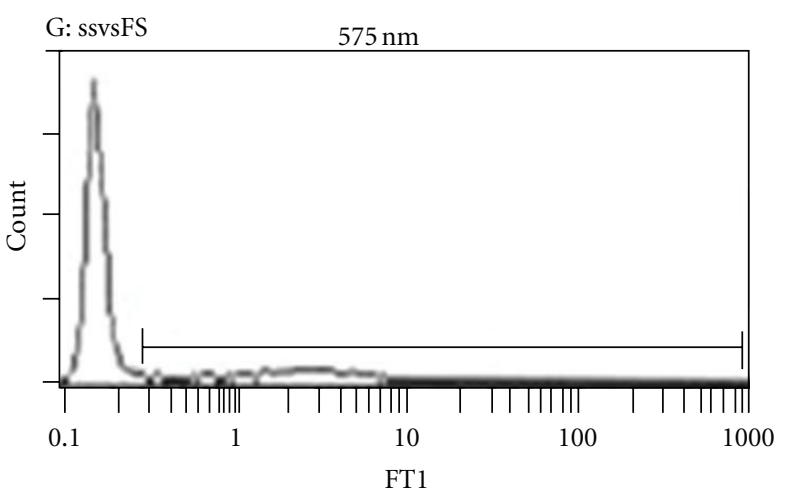

(b)

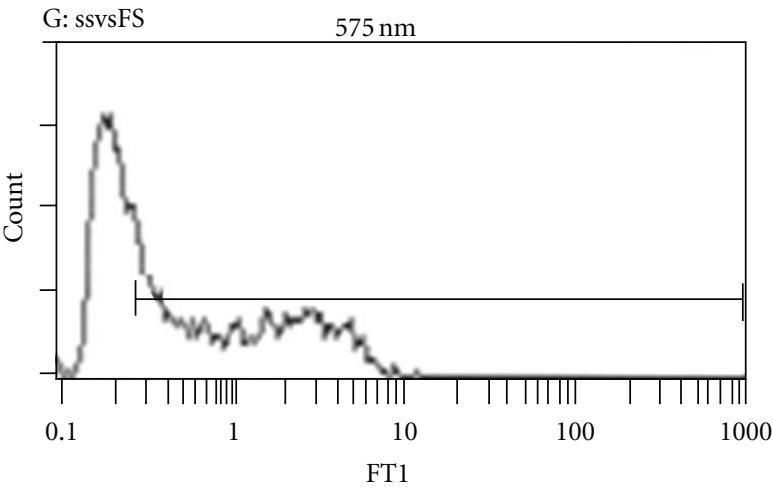

(d)

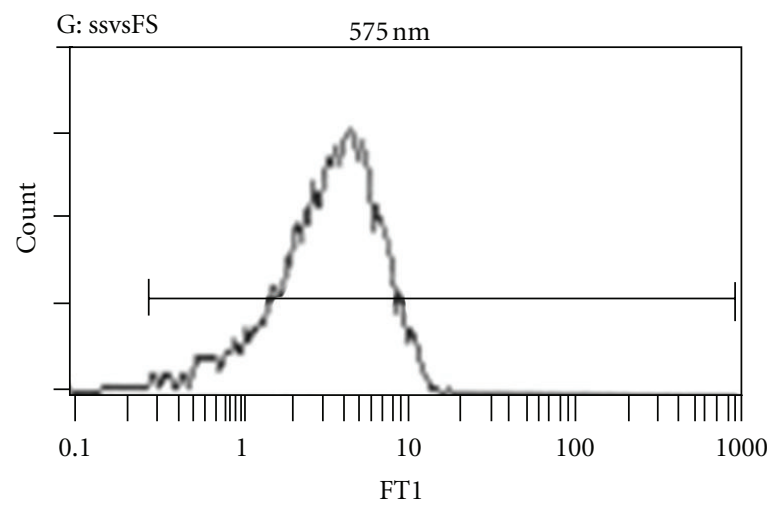

(e)

Figure 4: Effect on upregulation of IL-2R expression level on T cell surface of T $\alpha 13$. T lymphocytes from B6 mice spleen were cultured in the presence of ConA (b) or ConA plus standard synthesized T $\alpha 1$ (c), recombinant T $\alpha 1$ (d), and recombinant T $\alpha 1$ (3) (e), respectively. (a) The unstrained control. Data are representative of three experiments.

ConA, IL-2R expression level on $\mathrm{T}$ cell was upregulated by all these three molecules and $\mathrm{T} \alpha 13$ obtained strongest effect (Figure 4).

\section{Conclusions}

Trx-T $\alpha 13$ was expressed in E. coli as a soluble form and the real "natural" $\mathrm{T} \alpha 13$ (3) was conveniently purified by heat treatment and ion-exchange chromatography. As expected, the bioactivity of $\mathrm{T} \alpha 13$, was stronger than that of synthetic $\mathrm{T} \alpha 1$. Lower dose $(5 \mu \mathrm{g} / \mathrm{mL})$ of $\mathrm{T} \alpha 13$ apparently stimulated the proliferation of $\mathrm{T}$ lymphocytes compared with that of ZADAXIN $(40 \mu \mathrm{g} / \mathrm{mL})$. In addition, T $\alpha 13$ significantly upregulated IL-2R on T cell, which is very important for T cell activation and proliferation in vivo. The detailed mechanism for stronger effect of $\mathrm{T} \alpha 13$ and the pharmacokinetics of different tandem repeats are still under investigation.

\section{Acknowledgment}

This project was supported by the Natural Science Fund of China (Project no. 31000406). 


\section{References}

[1] R. I. MacDonald and E. V. Pozharski, "Free energies of urea and of thermal unfolding show that two tandem repeats of spectrin are thermodynamically more stable than a single repeat," Biochemistry, vol. 40, no. 13, pp. 3974-3984, 2001.

[2] J. Varea, J. L. Saiz, C. López-Zumel et al., "Do sequence repeats play an equivalent role in the choline-binding module of pneumococcal LytA amidase?" Journal of Biological Chemistry, vol. 275, no. 35, pp. 26842-26855, 2000.

[3] C. A. Buscaglia, J. Alfonso, O. Campetella, and A. C. C. Frasch, "Tandem amino acid repeats from Trypanosoma cruzi shed antigens increase the half-life of proteins in blood," Blood, vol. 93, no. 6, pp. 2025-2032, 1999.

[4] A. Schneider, A. Hemphill, T. Wyler, and T. Seebeck, "Large microtubule-associated protein of $\mathrm{T}$. brucei has tandemly repeated, near-identical sequences," Science, vol. 241, no. 4864, pp. 459-462, 1988.

[5] P. C. Cotrim, G. Paranhos-Baccala, M. R. Santos et al., "Organization and expression of the gene encoding an immunodominant repetitive antigen associated to the cytoskeleton of Trypanosoma cruzi," Molecular and Biochemical Parasitology, vol. 71, no. 1, pp. 89-98, 1995.

[6] A. L. Goldstein, T. L. Low, M. McAdoo et al., “Thymosin alpha 1: isolation and sequence analysis of an immunologically active thymic polypeptide," Proceedings of the National Academy of Sciences of the United States of America, vol. 74, pp. 725-729, 1977.

[7] T. L. K. Low and A. L. Goldstein, "The chemistry and biology of thymosin. II. Amino acid sequence analysis of thymosin $\alpha 1$ and polypeptide $\beta 1$," Journal of Biological Chemistry, vol. 254, no. 3, pp. 987-995, 1979.

[8] F. Salvati, G. Rasi, L. Portalone, A. Antilli, and E. Garaci, "Combined treatment with thymosin-alphal and low-dose interferon-alpha after ifosfamide in non-small cell lung cancer: a phaseII controlled trial," Anticancer Research, vol. 16, no. 2, pp. 10011004, 1996.

[9] S. Moscarella, G. Buzzelli, R. G. Romanelli et al., "Interferon and thymosin combination therapy in naive patients with chronic hepatitis C: preliminary results," Liver, vol. 18, no. 5, pp. 366369, 1998.

[10] F. Pica, M. Fraschetti, C. Matteucci, C. Tuthill, and G. Rasi, "High doses of thymosin alpha 1 enhance the anti-tumor efficacy of combination chemo-immunotherapy for murine B16 melanoma," Anticancer Research, vol. 18, no. 5, pp. 3571-3578, 1998.

[11] X. Xue, Z. Wang, Z. Yan, J. Shi, W. Han, and Y. Zhang, "Production and purification of recombinant human BLyS mutant from inclusion bodies," Protein Expression and Purification, vol. 42, no. 1, pp. 194-199, 2005.

[12] J. Kim, J. M. Park, and B. J. Lee, "HIGH-level expression and efficient purification of the antimicrobial peptide gaegurin 4 in E. coli," Protein and Peptide Letters, vol. 4, no. 6, pp. 391-396, 1997.

[13] Y. Chen, L. Zhao, G. Shen et al., "Expression and analysis of thymosin $\alpha 1$ concatemer in Escherichia coli," Biotechnology and Applied Biochemistry, vol. 49, no. 1, pp. 51-56, 2008.

[14] J. H. Shi, Y. T. Zhao, J. L. Wang, W. Han, Z. Yan, and Y. Q. Zhang, "Analysis of low molecular peptides by sodium dodecyl sulfatepolyacrylamide gel electrophoresis," Journal of the Fourth Military Medical University, vol. 21, no. 6, pp. 761-763, 2000. 

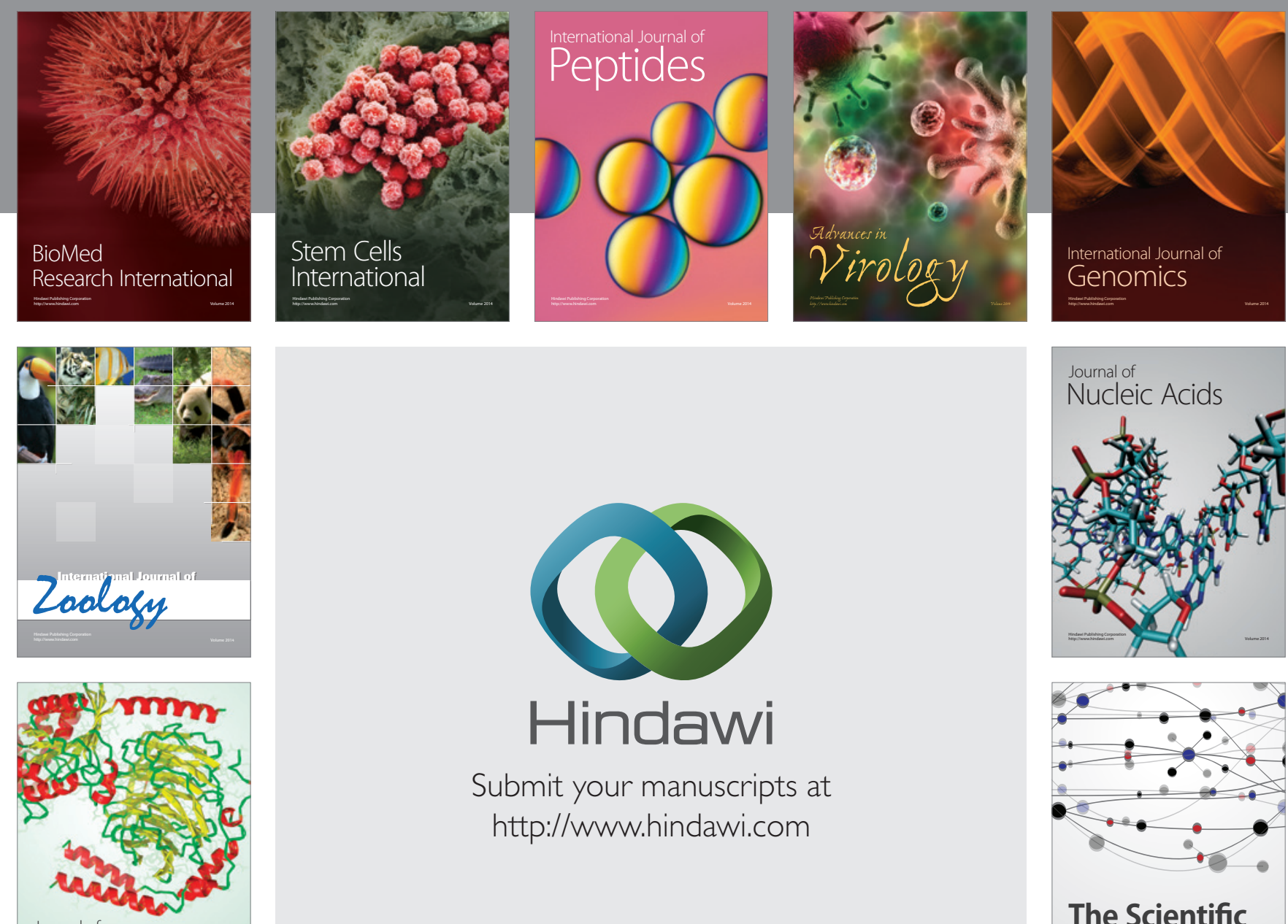

Submit your manuscripts at

http://www.hindawi.com

Journal of
Signal Transduction
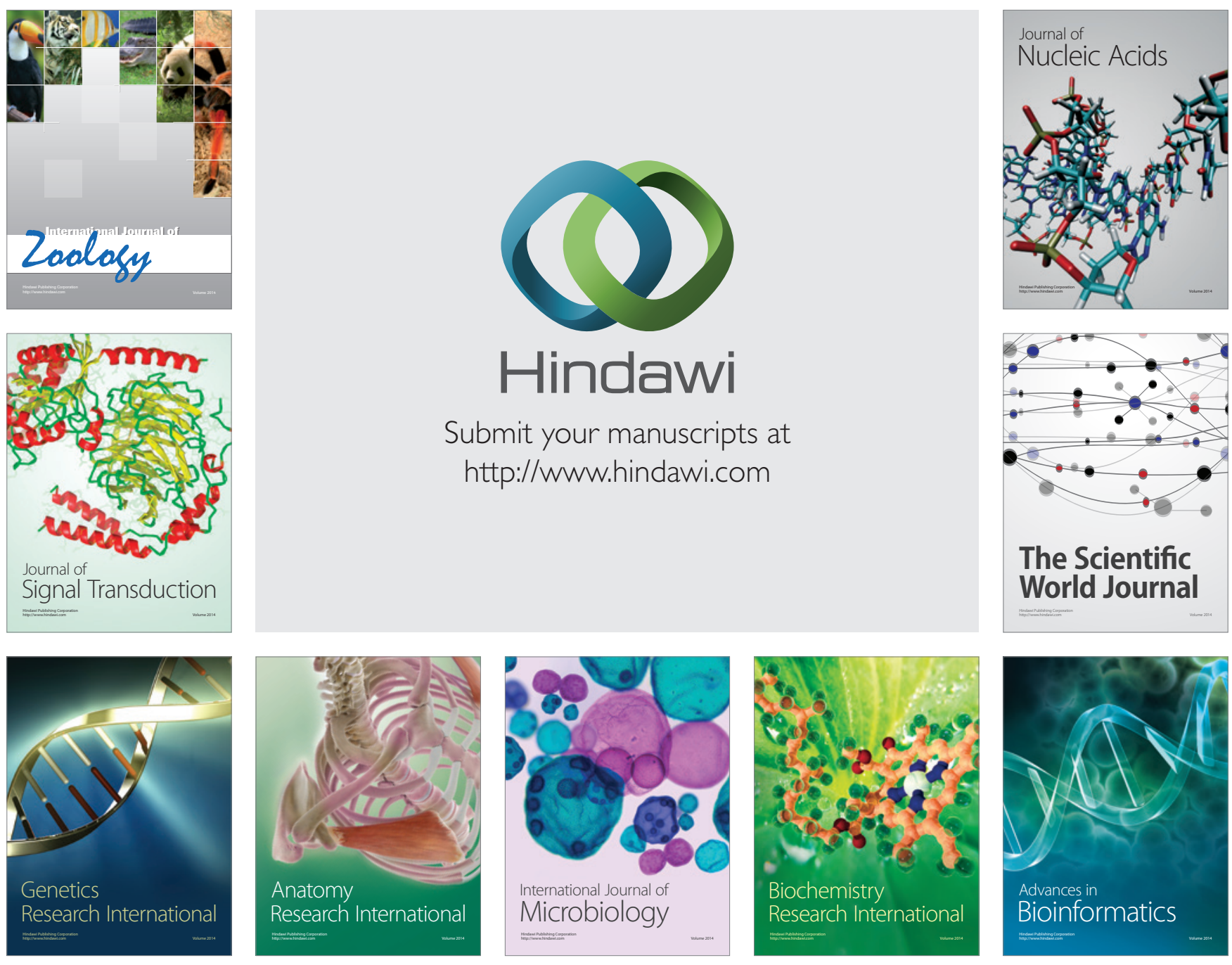

The Scientific World Journal
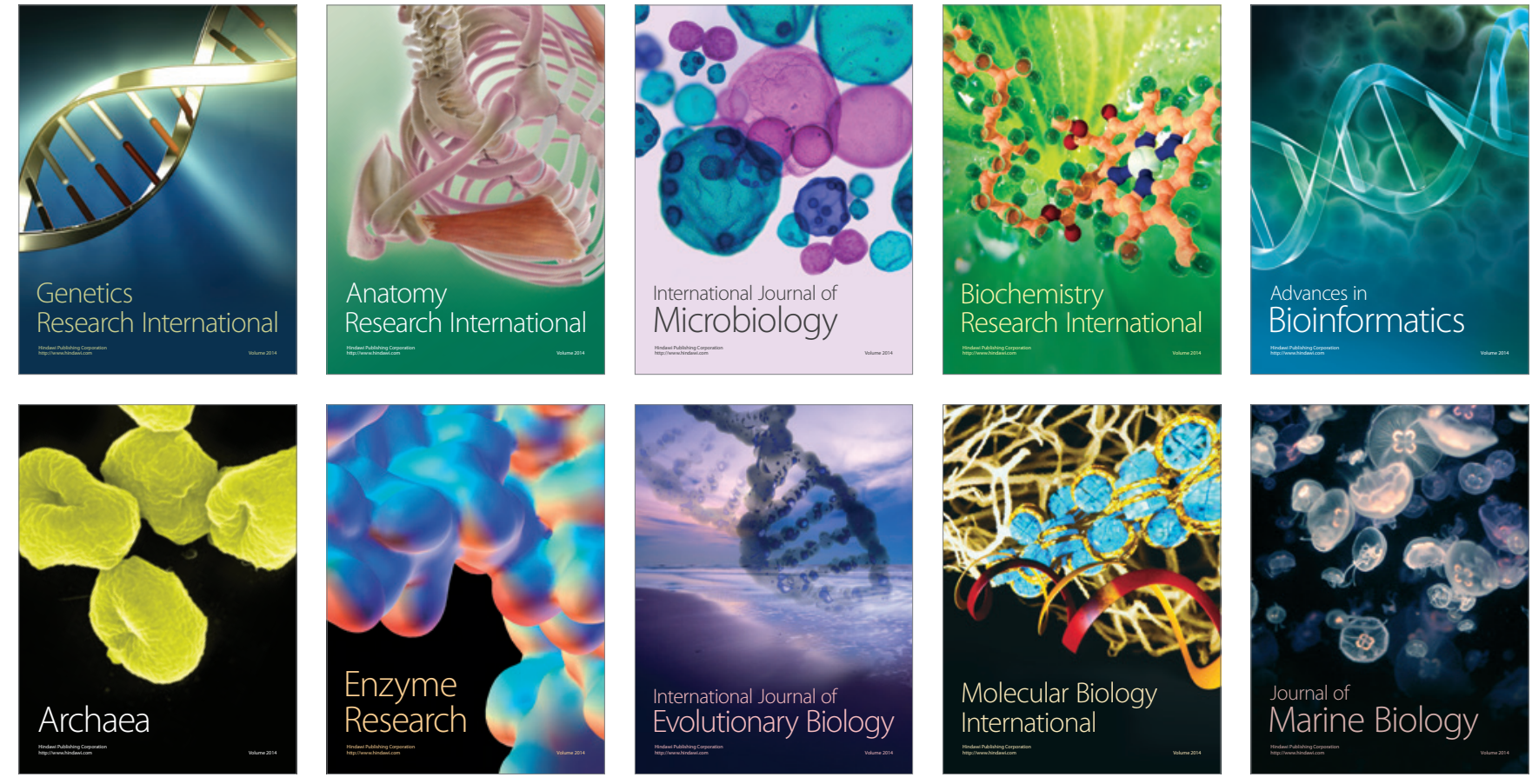